\title{
Diet enriched with cis9,trans11 CLA isomer regulates spleen proliferative immune response in rats
}

\author{
C. Ramírez-Santana ${ }^{1,2}$, A. Franch ${ }^{1,2}$, M. Castell ${ }^{1}$, M. Rivero ${ }^{3}$, M. Rodríguez-Palmero $^{3}$, C. Castellote $^{1,2}$ \\ and F. J. Pérez-Cano \\ ${ }^{1}$ Department of Physiology, Faculty of Pharmacy, University of Barcelona, Barcelona, Spain, ${ }^{2}$ CIBER in Epidemiology \\ and Public Health (CIBERESP), Spain and ${ }^{3}$ Research Department, Ordesa Group, Scientific Park of Barcelona, \\ Barcelona, Spain
}

Conjugated linoleic acid (CLA) seems to induce health effects in humans, but it has been suggested that it is the $c i s 9$, trans 11 ( $c 9, t 11$ ) CLA isomer that may be responsible for modulating the immune response ${ }^{(1)}$. The present study evaluates the effect of supplementing rats with an 80:20 mix of $c 9, t 11$ and $t 10, c 12$ CLA, respectively, on the capacity of generating polyclonal and antigen-specific immune responses.

A group of rats received CLA $(n=20)$ during gestation and suckling through dams fed 1\% CLA enriched diet and directly after weaning were fed same CLA dams diet until the adult age (15 weeks). Rats fed standard diet AIN-93G $(n=20)$ were used as reference group. At 9-week-old of age all animals were immunized with ovalbumin (OVA) and 6 weeks later, spleens were removed and splenocytes were isolated and cultured. Cell proliferation was assayed by addition of polyclonal stimulus (PMA/Io, 250 ng/ml) for $72 \mathrm{~h}$ and determined by an ELISA method based on $5^{\prime}$-bromo- $2^{\prime}$ deoxyuridine cell incorporation. Supernatant IL- 2 concentration after $24 \mathrm{~h}$ in same activation conditions were also quantified by ELISA. Specific proliferative response was evaluated by stimulating culture cells with OVA $(10 \mu \mathrm{g} / \mathrm{ml})$ for $96 \mathrm{~h}$. Anti-OVA Ig production was determined in spleen culture supernatants and sera by ELISA, whereas spleen anti-OVA $\mathrm{IgA}$-, IgG- and IgM-secreting cells (SC) were quantified by ELISPOT. Conventional ANOVA and post hoc comparisons were performed.

Splenocytes from animals which received CLA diet during all their life presented a lower $(\sim 10 \%)$ proliferative response after PMA/Io stimulation $(P<0.05)$, which was also correlated to IL-2 cytokine reduction. The down-regulatory effect was not due to spleen cell viability loss. However, splenocytes from animals fed CLA diet presented a significant increase $(\sim 110 \%)$ in proliferative response after antigen (OVA)-stimulation compared to that of reference animals $(P<0.05)$. OVA-immunized rats showed higher number of spleen antiOVA IgG- and IgM-SC than IgA-SC. This pattern was not affected by long-term dietary CLA, although a tendency to increase the number of anti-OVA IgA-SC was exhibited. No differences were observed for anti-OVA Ig production between both dietary groups.

These results demonstrate that, although long-term supplementation with $c 9, t 11$ CLA seems not to affect humoral response, it is able to down-regulate unspecific lymphocyte proliferative function and to enhance specific proliferation by immunocompetent cells.

1. Ramírez-Santana C, Castellote C, Castell M et al. (2009) J Nutr 139, 76-81. 